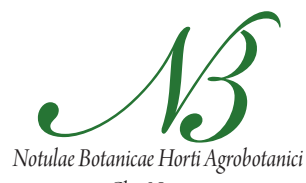

Cluj-Napoca

\title{
Determination of Tolerance Levels of Cotton Genotypes Obtained from F6-F7 Generation against Verticillium Wilt Disease Caused by Verticillium dabliae Kleb.
}

\author{
Oktay ERDOĞAN ${ }^{1}$, Emine KARADEMIR 2 , Çetin KARADEMIR ${ }^{2}$, Aydın UNAY ${ }^{3}$ \\ ${ }^{1}$ Cotton Research Station, 09800, Nazilli, Aydin, Turkey; oktaye@gmail.com \\ ${ }^{2}$ GAP International Agricultural Research and Training Center, 21100, Diyarbakır, Turkey \\ ${ }^{3}$ Adnan Menderes University, Faculty of Agriculture, Dept. of Field Crops, 09100, Aydin, Turkey
}

\begin{abstract}
The susceptibility of cotton genotypes obtained from $\mathrm{F}_{6}$ and $\mathrm{F}_{7}$ generations to Verticillium wilt (VW) disease (Verticillium dabliae Kleb.), was studied under artificial and natural infestation during 2009 and 2010 growing seasons at the Cotton Research Institute's, Nazilli, Aydın, Turkey. In this study, fifteen cotton breeding lines and two control varieties were used as plant material. During the cotton growing season, foliar disease index (FDI), vascular disease index (VDI) and pot disease index (PDI) were observed in addition to seed cotton yield and some fiber quality characteristics. According to the obtained results, disease severity (DS) values observed in pot experiments were higher than field trials. In terms of DS, 'Tamcot CD3H' x 'Tamcot Luxor-III', 'Maraş92' × 'Tamcot Sphinx' and 'Sayar 314' × 'Stoneville 453-I' breeding lines exhibited similar values with tolerant 'Carmen' variety. 'Tamcot CD3H' × 'Tamcot Sphinx', 'Sayar 314 × 'Stoneville 453-I', 'Sayar 314' × 'Stoneville 453-II' and 'Stoneville 453' × 'Tamcot Sphinx' breeding lines which gave similar DS values with tolerant 'Carmen' variety were statistically on the same group with 'Carmen' variety suitable to seed cotton yield. In this study, the disease tolerant breeding lines had higher fiber strength values than susceptible lines, the lowest fiber strength value obtained from 'Cukurova 1518' susceptible variety.
\end{abstract}

Keywords: cotton, disease intensity, tolerance, seed cotton yield, wilt

\section{Introduction}

Cotton is one of the most important strategic crops playing a key role in economic development of Turkey and all counties where it cultivated. In Turkey, totally 542.000 ha of Upland cotton was grown and 954.600 tons lint yield was produced in Southeastern Anatolia, Aegean, Çukurova and Antalya Regions (Anonymous, 2011).

Verticillium dabliae fungus causes diseases up to 400 plant species in many crops such as vegetables, leguminous plants, ornamentals, industrial plants, orchards and wild plants apart from cotton (Joaquim and Rowe, 1990). Yield losses due to VW in cotton were found 75\% in California, 8-10\% in Russia and 4\% in Syria (Bejarano-Alcazar et al., 1996). In China, VW index was determined between 3.61-28.30\% and rate of infected plants were changed from $0.6-60 \%$ (Wu and Wu, 1997). It was reported that the disease causes yield losses with average $3.1 \%$ per year on 10 years period in the USA (Devay, 2001). Control of VW is difficult under intensive cropping systems. Chemical control is not an effective method, thus one of the most effective methods for controlling disease is using resistant varieties (El-Zik, 1985; Erdoğan et al., 2006; Wilhelm et al., 1974).
Allen and Lonergen (1998) observed that VW was the most common and significant disease in Australia, a few resistant cotton varieties have been developed by CSIRO since 1989 and these varieties had been adapted and grown. Kurt and Bicici (1998) reported that 'Deltapine 15/21', 'Maraş 92', 'Sayar 314' and 'Erşan 92' varieties have high susceptibility to T-1 pathotype. 'Çukurova 1518 ' variety has very low resistant to non-defoliating pathotype in field. All varieties except for 'DPL 15/21', 'Çukurova 1518' and 'Sayar 314' varieties were middle or high resistant to non-defoliating pathotype. In 2000, Mert et al. (2001) tested 13 cotton genotypes to Verticillium wilt in Adana and Hatay, they evaluated these cotton genotypes according to both foliar symptoms and vascular symptoms. The result indicated that 'PAUM 400, '401', '402,' ‘403', '404', '405', '406' breeding lines and 'Nazilli 143' variety were resistant, while 'Çukurova 1518 ' was susceptible. Galanopoulo (2006) found that the cotton varieties belong to Gossypium hirsutum were not exactly immune, the reactions of these cultivars varied to VW. The objective of this study was to determine susceptibilities of cotton breeding lines against VW disease (V.dabliae Kleb.). 


\section{Materials and methods}

\section{Source of Plant materials and pathogen used}

The study was carried out during 2009 and 2010 growing seasons at Cotton Res. Inst. fields, Nazilli, Aydın, Turkey. Two cotton cultivars were used as control. $V$. dabliaetolerant Upland control cultivar 'Carmen' (Bayer Crop Science AG, Leverkusen, Germany), and sensitive local control variety 'Çukurova 1518' (Çukurova Agricultural Research Institute, Adana, Turkey), and 15 breeding lines (GAP International Agricultural Research and Training Center, Diyarbakır, Turkey) totally 17 genotypes were used as experiment material. Also, highly virulent $V$. dabliae strain (VD-11, non-defoliating pathotype) was isolated previously from wilted cotton plants (Erdoğan, 2007) and VD-11 isolate was used for artificial inoculation as described by (Erdoğan, 2009). Inoculum of VD-11 isolate was prepared by growing on plates containing Potato dextrose agar medium (PDA) the plates were incubated at $24^{\circ} \mathrm{C}$. Two weeks later conidia were gently collected, suspended by washing with sterile water. The concentration of conidia was determined using a hemacytometer and appropriate dilutions were made to obtain a conidia suspension with concentration of $3 \times 10^{7}$ conidia $\mathrm{mL}^{-1}$ and used for inoculation

\section{Greenhouse trials}

Plants were inoculated with spore suspension by the stem-injection (Bejarano-Alcazar et al., 1996) in pot experiment which was carried out as randomized plot with four replicates under greenhouse condition $\left(\right.$ at $24 \pm 1^{\circ} \mathrm{C}$ ). For this purpose, plastic pots which has been autoclaved at $121^{\circ} \mathrm{C}$ for $1 \mathrm{~h}$ filled with sterilized soil, disinfested $(1 \%$ $\mathrm{NaOCl}$ for $2.5 \mathrm{~min}$ ) germinated seeds were sown in pots in different 5 points. Three of seedlings were removed by thinning two seedlings remained in each pot at cotyledon leaves period. Plants at the six-true-leaf stage were woundinoculated by puncturing the stem at the first internodes above the soil line with a 22 -gauge needle through a 5 - $\mu$ l drop of the conidial suspension (Bugbee and Presley, 1967). Control plants were inoculated with sterile water and 14 days after inoculation, plants were rated for leaf disease severity using a rating scale for 0-3 (Hanson, 2000). Results were combined for all leaves to determine a severity rating for the plant (PDI).

\section{Field trials}

Field trial was conducted at natural infested experimental area with the non- defoliating pathotype between 2009 and 2010 in Institute field. The inoculum density of pathogen in naturally infested field was determined as $69 \mathrm{CFU}$ (microsclerotia ${ }^{-1}$ ) (Erdoğan et al., 2011). The experimental design were established in randomized complete block design with four replications and each plot consisted of four rows of $12 \mathrm{~m}$ length, between and within the rows, spacing was $70 \mathrm{~cm}$ and $20-25 \mathrm{~cm}$ respectively. Initial soil analysis showed that experimental area had claying soil, slight alkali, low salinity and rich for lime, organic matter, potassium and phosphorous. Sowing was made with combine cotton drilling machine. First year, planting was performed on 19 May 2009, the second year on 15 May 2010; all plots were treated with 20-20-0 composite fertilizer providing $60 \mathrm{~kg} \mathrm{ha}^{-1} \mathrm{~N}$ and $60 \mathrm{~kg} \mathrm{ha}^{-1} \mathrm{P}_{2} \mathrm{O}_{5}$. Just before flowering, $60 \mathrm{~kg} \mathrm{ha}^{-1} \mathrm{~N}$ (as 33\% ammonium nitrate) was applied to the trial as an additional $\mathrm{N}$ source.

Observations of wilt and foliar diseases were taken from the middle 2 rows of each plot with all of the plants on leaves at 5-10\% (FDI-1) and 50-60\% (FDI-2) cotton boll opening stage (Unal and Aydın, 1980) and stems following the last harvesting by hand. 0-3 scale was used for observations of diseases (VDI) (Buchenauer and Erwin, 1976). Disease rates were calculated and obtained data were subjected to Arcsin for transformation (Karman, 1971). Statistical analyses were performed using JMP 5.0.1 statistical software and the means were grouped by means of the LSD $(p=0.05)$ test. Also, seed cotton yield (SCY), ginning percentage (GP), fiber fineness (FF), fiber length (FL), and fiber strength (FS) were recorded from two rows of each plot.

\section{Results}

The mean values obtained from the investigated characteristics, regarding FDI-1 and FDI-2, VDI and PDI are presented in Tab. 1. Mean values of SCY, and GP are presented in Tab. 2; mean values of FL, FF and FS are presented in Tab. 3. As seen in Tab. 1, in the statistical analysis made pursuant to disease severity values, genotype was found significant on the range of FDI-1 and FDI-2, VDI and PDI in $\mathrm{F}_{6}$ and $\mathrm{F}_{7}$ generations. On the FDI- 1 of these generations, 'Carmen' control variety and 'Tamcot $\mathrm{CD} 3 \mathrm{H}^{\prime} \times$ 'Tamcot Luxor-III' that was tolerant showed the lowest disease severity value with $0.50-0.55$ index and was followed by 'Maraş92' × 'Tamcot Sphinx' (0.55-0.58) and 'Sayar 314' × 'Stoneville 453-I' (0.55-0.62) and as for 'Çukurova 1518 ' control variety, which is susceptible the highest disease severity value was seen. On the FDI-2, the lowest disease severity $(0.55-0.73)$ was ascertained in tolerant 'Carmen' control variety and 'Sayar314' × 'TamcotHQ95' (0.86-1.00) and 'Tamcot CD3H' × 'Tamcot Sphinx' (0.89-0.93) followed that, and the highest disease severity was identified in susceptible 'Çukurova 1518' control variety. On VDI, tolerant 'Carmen' control variety showed the lowest disease severity value with $0.62-0.87$ index and was followed by 'Tamcot CD3H' $\times$ 'Tamcot Luxor-I' (0.93-1.05) and 'Tamcot CD3H' $\times$ 'Tamcot Luxor-III' (0.96-1.00) and the highest disease severity value (2.09-2.11) was identified in susceptible 'Çukurova 1518 ' control variety. On PDI experiments, tolerant 'Carmen' control variety indicated the lowest disease severity value $(35.9 \%-39.4 \%)$ and was followed by 'Sayar 314 ' $\times$ 'Tamcot Luxor' (37.7\% - 41.3\%). The highest disease severity value $(84.3 \%-84.9 \%)$ was identified in susceptible 'Çukurova 1518' control variety and 'Stoneville453' $\times$ 'Tamcot Sphinx-III’ (71.8\%-72.0\%) (Tab. 1.) 
186

Results from the analysis of variance for SCY and GP are given in Tab. 2. As seen in the Tab. 2, there were significant differences among genotypes. While 'TamcotCD3H' $\times$ 'Tamcot Sphinx' (4950.67 $\mathrm{kg} \mathrm{ha}^{-1}$ ) and 'Sayar314' $\times$ 'Stoneville453-I' (4770.67 kg ha-1) and 'Sayar314' $\times$ 'Stoneville453-II' ( $4730.69 \mathrm{~kg} \mathrm{ha}^{-1}$ ) were the first in terms

of yield and located in the same group in $\mathrm{F}_{6}$ generation, 'Carmen' ( $4210.62 \mathrm{~kg} \mathrm{ha}^{-1}$ ) and 'Stoneville453' $\times$ 'Tamcot Sphinx' (4030.62 $\mathrm{kg} \mathrm{ha}^{-1}$ ) were the first in terms of yield and located in the same group in the $\mathrm{F}_{7}$ generation. While the lowest yield was also identified in 'Çukurova 1518 ' control variety $\left(3900.29 \mathrm{~kg} \mathrm{ha}^{-1}\right)$ and 'Sayar314' $\times$ 'Tam-

Tab. 1. Mean values of foliar disease index (FDI-1, FDI-2), vascular disease index (VDI), pot diseases index (PDI) in $\mathrm{F}_{6}-\mathrm{F}_{7}$ generation (2009 and 2010)

\begin{tabular}{|c|c|c|c|c|c|c|c|c|}
\hline \multirow{2}{*}{ Genotype } & \multicolumn{4}{|c|}{ F6 Generation } & \multicolumn{4}{|c|}{ F7 Generation } \\
\hline & FDI-1 & FDI-2 & VDI & PDI (\%) & FDI-1 & FDI-2 & VDI & PDI (\%) \\
\hline 'Maraş92' × 'Sayar314' & $0.64 c-e$ & $1.02 \mathrm{~b}-\mathrm{e}$ & $1.19 \mathrm{~b}$ & $62.5 \mathrm{bc}$ & $0.63 \mathrm{ef}$ & $1.09 \mathrm{~d}$ & $1.20 \mathrm{de}$ & $61.1 \mathrm{bc}$ \\
\hline 'Maraş92' × 'TamcotSphinx' & $0.55 \mathrm{de}$ & $0.90 \mathrm{c}-\mathrm{e}$ & $1.17 \mathrm{~b}$ & $42.0 \mathrm{e}-\mathrm{h}$ & $0.58 \mathrm{fg}$ & $1.19 \mathrm{~b}$ & $1.30 \mathrm{~b}$ & $43.0 \mathrm{e}-\mathrm{h}$ \\
\hline 'Sayar314' × 'Stoneville453-I' & $0.55 \mathrm{de}$ & $0.94 \mathrm{c}-\mathrm{e}$ & $1.02 \mathrm{~b}$ & $48.3 \mathrm{~d}-\mathrm{h}$ & $0.62 \mathrm{ef}$ & $1.00 \mathrm{e}$ & $1.12 \mathrm{e}-\mathrm{g}$ & $48.8 \mathrm{~d}-\mathrm{g}$ \\
\hline 'Sayar314' × 'Stoneville453-II' & $0.59 \mathrm{c}-\mathrm{e}$ & $1.03 \mathrm{~b}-\mathrm{e}$ & $0.92 \mathrm{bc}$ & $50.0 \mathrm{~d}-\mathrm{g}$ & $0.64 \mathrm{ef}$ & $1.10 \mathrm{~cd}$ & $1.20 \mathrm{c}-\mathrm{e}$ & $50.3 \mathrm{~d}-\mathrm{g}$ \\
\hline 'Sayar 314' x 'TamcotCD3H' & $0.61 \mathrm{c}-\mathrm{e}$ & $1.03 \mathrm{~b}-\mathrm{e}$ & $0.97 \mathrm{~b}$ & $51.1 \mathrm{c}-\mathrm{f}$ & $0.65 \mathrm{ef}$ & $1.10 \mathrm{~cd}$ & $1.22 \mathrm{~b}-\mathrm{d}$ & $51.3 \mathrm{~d}-\mathrm{g}$ \\
\hline 'Sayar 314' × 'TamcotHQ95' & $0.61 \mathrm{c}-\mathrm{e}$ & $0.86 \mathrm{e}$ & $1.02 \mathrm{~b}$ & $57.6 \mathrm{~cd}$ & $0.61 \mathrm{ef}$ & $1.00 \mathrm{e}$ & $1.11 \mathrm{fg}$ & $57.2 \mathrm{c}-\mathrm{e}$ \\
\hline 'Sayar 314’ × 'Tamcot Luxor' & $0.72 b-e$ & $1.01 \mathrm{~b}-\mathrm{e}$ & $1.13 \mathrm{~b}$ & $37.7 \mathrm{gh}$ & $0.68 \mathrm{de}$ & $1.09 \mathrm{~cd}$ & $1.18 \mathrm{~d}-\mathrm{f}$ & $41.3 \mathrm{~d}-\mathrm{h}$ \\
\hline 'Stoneville453' x 'TamcotSphinx' & $0.78 b-d$ & $1.02 \mathrm{~b}-\mathrm{e}$ & $1.03 \mathrm{~b}$ & $55.6 \mathrm{~cd}$ & $0.69 \mathrm{c}-\mathrm{e}$ & $1.14 b-d$ & $1.25 b-d$ & $56.3 \mathrm{c}-\mathrm{f}$ \\
\hline 'Stoneville453' × 'TamcotSphinx-I' & $0.80 \mathrm{bc}$ & $1.13 b c$ & $1.01 \mathrm{~b}$ & $45.3 \mathrm{~d}-\mathrm{h}$ & $0.78 b$ & $1.19 \mathrm{~b}$ & $1.31 \mathrm{~b}$ & $47.3 \mathrm{e}-\mathrm{h}$ \\
\hline 'Stoneville453’ × 'TamcotSphinx-II' & $0.73 \mathrm{~b}-\mathrm{e}$ & $0.93 \mathrm{c}-\mathrm{e}$ & $1.22 b$ & $39.1 \mathrm{f}-\mathrm{h}$ & $0.67 \mathrm{de}$ & $0.87 f$ & $1.04 \mathrm{gh}$ & $42.4 \mathrm{f}-\mathrm{h}$ \\
\hline 'Stoneville453’ × ‘TamcotSphinx-III’ & $0.89 \mathrm{~b}$ & $1.04 \mathrm{~b}-\mathrm{e}$ & $1.06 \mathrm{~b}$ & $71.8 \mathrm{~b}$ & $0.77 \mathrm{bc}$ & $1.17 \mathrm{bc}$ & $1.29 \mathrm{bc}$ & $72.0 \mathrm{~b}$ \\
\hline 'TamcotCD3H' × 'TamcotSphinx' & $0.62 \mathrm{c}-\mathrm{e}$ & $0.89 \mathrm{de}$ & $1.13 \mathrm{~b}$ & $53.8 \mathrm{c}-\mathrm{e}$ & $0.68 \mathrm{de}$ & $0.93 \mathrm{ef}$ & $1.07 \mathrm{gh}$ & $57.2 \mathrm{~cd}$ \\
\hline 'TamcotCD3H' × ‘TamcotLuxor-I’ & $0.63 \mathrm{c}-\mathrm{e}$ & $1.11 \mathrm{~b}-\mathrm{d}$ & $0.93 \mathrm{bc}$ & $41.8 \mathrm{e}-\mathrm{h}$ & $0.64 \mathrm{ef}$ & $0.97 \mathrm{e}$ & $1.05 \mathrm{gh}$ & $45.9 \mathrm{gh}$ \\
\hline 'TamcotCD3H' × 'TamcotLuxor-II' & $0.76 b-d$ & $1.22 \mathrm{~b}$ & $1.03 \mathrm{~b}$ & $45.7 \mathrm{~d}-\mathrm{h}$ & $0.76 \mathrm{~b}-\mathrm{d}$ & $1.13 b-d$ & $1.22 \mathrm{~b}-\mathrm{d}$ & $48.5 \mathrm{f}-\mathrm{h}$ \\
\hline 'TamcotCD3H' × 'TamcotLuxor-III' & $0.50 \mathrm{e}$ & $0.96 \mathrm{c}-\mathrm{e}$ & $0.96 \mathrm{bc}$ & $51.0 \mathrm{c}-\mathrm{g}$ & $0.57 \mathrm{fg}$ & $0.88 \mathrm{f}$ & $1.00 \mathrm{~h}$ & $53.8 \mathrm{c}-\mathrm{f}$ \\
\hline ‘Carmen’ & $0.50 \mathrm{e}$ & $0.55 f$ & $0.62 \mathrm{c}$ & $35.9 \mathrm{~h}$ & $0.50 \mathrm{~g}$ & $0.73 \mathrm{~g}$ & $0.87 \mathrm{i}$ & $39.4 \mathrm{~h}$ \\
\hline 'Çukurova 1518' & $1.79 \mathrm{a}$ & $2.11 \mathrm{a}$ & $2.09 \mathrm{a}$ & $84.3 \mathrm{a}$ & $1.84 \mathrm{a}$ & $2.00 \mathrm{a}$ & $2.11 \mathrm{a}$ & $84.9 \mathrm{a}$ \\
\hline Mean & 0.72 & 1.04 & 1.09 & 51.4 & 0.73 & 1.09 & 1.21 & 52.9 \\
\hline & * & * & * & $*$ & * & $*$ & * & * \\
\hline LSD & 0.133 & 0.131 & 0.192 & 8.602 & 0.052 & 0.044 & 0.048 & 7.860 \\
\hline
\end{tabular}

${ }^{*}$ Different letters between genotypes denote significant differences (LSD test, $p<0.05$ )

Tab. 2. Mean values of seed cotton yield (SCY) and ginning percentage (GP) in $F_{6}-F_{7}$ generation (2009 and 2010)

\begin{tabular}{|c|c|c|c|c|}
\hline \multirow{2}{*}{ Genotype } & \multicolumn{2}{|c|}{ F6 Generation } & \multicolumn{2}{|c|}{ F7 Generation } \\
\hline & $\operatorname{SCY}\left(\mathrm{kg} \mathrm{ha}^{-1}\right)$ & GP (\%) & $\operatorname{SCY}\left(\mathrm{kg} \mathrm{ha}^{-1}\right)$ & GP (\%) \\
\hline 'Maraş92' × 'Sayar314' & $4320.83 a-c$ & $41.65 a$ & $3840.23 \mathrm{abc}$ & $40.50 \mathrm{a}$ \\
\hline 'Maraş92' × 'TamcotSphinx' & $4580.92 \mathrm{a}-\mathrm{c}$ & $37.40 \mathrm{gh}$ & $3800.34 b-d$ & $37.10 \mathrm{gh}$ \\
\hline Sayar314 × 'Stoneville453-I' & $4770.67 \mathrm{ab}$ & $40.10 \mathrm{bc}$ & $3680.38 b-e$ & $39.20 \mathrm{~b}-\mathrm{d}$ \\
\hline 'Sayar314' × 'Stoneville453-II' & $4730.69 \mathrm{ab}$ & $39.77 \mathrm{c}$ & $3980.59 \mathrm{a}-\mathrm{c}$ & $39.30 \mathrm{a}-\mathrm{d}$ \\
\hline 'Sayar 314' × 'TamcotCD3H' & $3900.74 \mathrm{c}$ & $39.25 c-e$ & $3930.21 \mathrm{a}-\mathrm{c}$ & $39.00 \mathrm{c}-\mathrm{e}$ \\
\hline 'Sayar 314’ × 'TamcotHQ95’ & $4520.33 a-c$ & $37.67 \mathrm{gh}$ & $3960.74 a-c$ & $38.10 \mathrm{~d}-\mathrm{h}$ \\
\hline 'Sayar 314’ x 'Tamcot Luxor' & $4260.70 a-c$ & $40.02 \mathrm{bc}$ & $3940.26 a-c$ & $38.50 \mathrm{c}-\mathrm{f}$ \\
\hline 'Stoneville 453 ' × 'TamcotSphinx' & $4020.73 b c$ & $38.10 \mathrm{e}-\mathrm{g}$ & $4030.62 \mathrm{ab}$ & $36.80 \mathrm{~h}$ \\
\hline 'Stoneville 453' × 'TamcotSphinx-I' & $4300.03 a-c$ & $39.22 \mathrm{c}-\mathrm{e}$ & $3840.02 \mathrm{a}-\mathrm{c}$ & $38.30 \mathrm{c}-\mathrm{g}$ \\
\hline 'Stoneville453’ × 'TamcotSphinx-II' & $4180.93 a-c$ & $37.50 \mathrm{gh}$ & $3350.87 \mathrm{e}$ & $37.90 \mathrm{e}-\mathrm{h}$ \\
\hline 'Stoneville453' × 'TamcotSphinx-III' & $4280.18 \mathrm{a}-\mathrm{c}$ & $38.52 \mathrm{~d}-\mathrm{g}$ & $3590.32 c-e$ & $37.30 \mathrm{f}-\mathrm{h}$ \\
\hline 'TamcotCD3H' × 'TamcotSphinx' & $4950.67 a$ & $37.87 \mathrm{f}-\mathrm{h}$ & $3660.37 b-e$ & $37.10 \mathrm{gh}$ \\
\hline 'TamcotCD3H' × 'TamcotLuxor-I' & $4100.02 b c$ & $36.82 \mathrm{~h}$ & $3900.37 \mathrm{a}-\mathrm{c}$ & $36.80 \mathrm{~h}$ \\
\hline ‘TamcotCD3H’ × 'TamcotLuxor-II’ & $4180.88 \mathrm{a}-\mathrm{c}$ & $38.92 \mathrm{c}-\mathrm{f}$ & $3600.74 c-e$ & $37.40 \mathrm{f}-\mathrm{h}$ \\
\hline ‘TamcotCD3H’ × ‘TamcotLuxor-III’ & $4160.11 a-c$ & $39.52 \mathrm{~cd}$ & $3810.89 \mathrm{a}-\mathrm{c}$ & $38.90 c-e$ \\
\hline ‘Carmen’ & $4410.35 a-c$ & $41.15 \mathrm{ab}$ & $4210.62 \mathrm{a}$ & $40.40 \mathrm{ab}$ \\
\hline 'Çukurova 1518' & $3900.29 c$ & $39.82 \mathrm{c}$ & $3400.53 \mathrm{de}$ & $39.40 a-c$ \\
\hline Mean & 4320.70 & 39.00 & 3800.00 & 38.30 \\
\hline $\mathrm{F}_{\text {(gcnotype) }}$ & $*$ & $*$ & $*$ & * \\
\hline LSD & 80.990 & 1.946 & 40.930 & 1.243 \\
\hline
\end{tabular}

${ }^{*}$ Different letters between genotypes denote significant differences (LSD test, $p<0.05$ ) 
Tab. 3. Mean values of fiber fineness (FF), fiber length (FL) and fiber strength (FS) in $\mathrm{F}_{6}-\mathrm{F}_{7}$ generation (2009 and 2010)

\begin{tabular}{|c|c|c|c|c|c|c|}
\hline \multirow{2}{*}{ Genotype } & \multicolumn{3}{|c|}{ F6 Generation } & \multicolumn{3}{|c|}{ F7 Generation } \\
\hline & $\mathrm{FF}$ (mic.) & $\mathrm{FL}(\mathrm{mm})$ & FS $\left(\mathrm{g} \mathrm{tex}^{-1}\right)$ & $\mathrm{FF}$ (mic.) & $\mathrm{FL}(\mathrm{mm})$ & FS $\left(\mathrm{g} \mathrm{tex}^{-1}\right)$ \\
\hline 'Maraş92' × 'Sayar314' & $4.76 \mathrm{~b}-\mathrm{e}$ & $29.08 \mathrm{c}-\mathrm{g}$ & $30.57 b$ & $4.90 \mathrm{~b}-\mathrm{d}$ & $29.92 b c$ & $33.20 \mathrm{ab}$ \\
\hline 'Maraş92' × 'TamcotSphinx' & $4.74 \mathrm{c}-\mathrm{e}$ & $30.12 b c$ & $33.15 \mathrm{a}$ & $4.82 \mathrm{c}-\mathrm{e}$ & $29.09 \mathrm{c}-\mathrm{e}$ & $32.47 a-c$ \\
\hline 'Sayar314' × 'Stoneville453-I' & $4.39 \mathrm{~g}$ & $31.11 \mathrm{ab}$ & $32.70 \mathrm{a}$ & $4.68 \mathrm{de}$ & $30.47 \mathrm{ab}$ & $33.27 \mathrm{ab}$ \\
\hline 'Sayar314' × 'Stoneville453-II' & $4.94 a-c$ & $29.67 \mathrm{c}-\mathrm{e}$ & $30.60 \mathrm{~b}$ & $5.19 \mathrm{a}$ & $30.12 b c$ & $32.60 \mathrm{a}-\mathrm{c}$ \\
\hline 'Sayar 314’ × 'TamcotCD3H' & $4.57 \mathrm{e}-\mathrm{g}$ & $29.55 \mathrm{c}-\mathrm{f}$ & $30.35 b$ & $4.87 \mathrm{c}-\mathrm{e}$ & $29.86 \mathrm{bc}$ & $32.30 \mathrm{a}-\mathrm{d}$ \\
\hline 'Sayar 314' × 'TamcotHQ95' & $4.49 \mathrm{fg}$ & $29.78 \mathrm{~cd}$ & $30.08 b$ & $4.70 \mathrm{de}$ & $29.74 \mathrm{bc}$ & $31.22 \mathrm{a}-\mathrm{d}$ \\
\hline 'Sayar 314’ × 'Tamcot Luxor' & $4.57 \mathrm{e}-\mathrm{g}$ & $29.37 \mathrm{c}-\mathrm{f}$ & $30.35 b$ & $4.73 \mathrm{de}$ & $29.45 b-d$ & $30.70 \mathrm{~cd}$ \\
\hline Stoneville 453 x 'TamcotSphinx' & $5.04 \mathrm{a}$ & $28.69 \mathrm{~d}-\mathrm{g}$ & $29.73 b$ & $5.24 \mathrm{a}$ & $27.99 \mathrm{fg}$ & $30.95 b-d$ \\
\hline Stoneville $453 \times$ × 'TamcotSphinx-I' & $4.85 a-d$ & $29.06 \mathrm{c}-\mathrm{g}$ & $29.88 b$ & $4.65 \mathrm{de}$ & $28.58 \mathrm{~d}-\mathrm{g}$ & $30.95 b-d$ \\
\hline 'Stoneville453’ × 'TamcotSphinx-II' & $4.75 b-e$ & $28.13 \mathrm{~g}$ & $30.45 b$ & $4.84 \mathrm{c}-\mathrm{e}$ & $28.55 \mathrm{~d}-\mathrm{g}$ & $32.20 \mathrm{a}-\mathrm{d}$ \\
\hline 'Stoneville453’ x ‘TamcotSphinx-III’ & $4.80 \mathrm{a}-\mathrm{e}$ & $29.15 \mathrm{c}-\mathrm{g}$ & $30.35 b$ & $4.84 \mathrm{c}-\mathrm{e}$ & $28.07 \mathrm{e}-\mathrm{g}$ & $31.45 \mathrm{a}-\mathrm{d}$ \\
\hline 'TamcotCD3H’ × 'TamcotSphinx' & $4.99 \mathrm{ab}$ & $29.82 \mathrm{~cd}$ & $30.50 \mathrm{~b}$ & $4.86 \mathrm{c}-\mathrm{e}$ & $29.88 \mathrm{bc}$ & $31.95 \mathrm{a}-\mathrm{d}$ \\
\hline ‘TamcotCD3H’ × 'TamcotLuxor-I’ & $5.02 \mathrm{a}$ & $28.41 \mathrm{fg}$ & $30.30 \mathrm{~b}$ & $5.15 \mathrm{ab}$ & $28.05 \mathrm{e}-\mathrm{g}$ & $31.70 \mathrm{a}-\mathrm{d}$ \\
\hline 'TamcotCD3H' × 'TamcotLuxor-II' & $4.78 \mathrm{~b}-\mathrm{e}$ & $28.82 \mathrm{~d}-\mathrm{g}$ & $29.22 b c$ & $4.78 \mathrm{de}$ & $29.08 \mathrm{c}-\mathrm{f}$ & $31.25 \mathrm{a}-\mathrm{d}$ \\
\hline ‘TamcotCD3H’ × ‘TamcotLuxor-III’ & $4.76 b-e$ & $28.53 \mathrm{e}-\mathrm{g}$ & $30.55 b$ & $4.70 \mathrm{de}$ & $27.98 \mathrm{~g}$ & $32.55 \mathrm{a}-\mathrm{c}$ \\
\hline 'Carmen' & $4.78 \mathrm{~b}-\mathrm{e}$ & $31.44 \mathrm{a}$ & $32.65 a$ & $4.62 \mathrm{e}$ & $31.22 \mathrm{a}$ & $33.40 \mathrm{a}$ \\
\hline 'Çukurova 1518' & $4.68 \mathrm{~d}-\mathrm{f}$ & $29.38 c-f$ & $27.80 \mathrm{c}$ & $5.05 \mathrm{a}-\mathrm{c}$ & $29.35 \mathrm{~cd}$ & $30.07 \mathrm{~d}$ \\
\hline Mean & 4.80 & 29.40 & 30.50 & 4.90 & 29.30 & 31.90 \\
\hline$F_{\text {(genotypc) }}$ & * & * & * & * & * & $*$ \\
\hline LSD & 0.235 & 1.212 & 1.652 & 0.259 & 1.099 & 2.366 \\
\hline
\end{tabular}

${ }^{*}$ Different letters between genotypes denote significant differences (LSD test, $p<0.05$ )

$\operatorname{cotCD} 3 \mathrm{H}^{\prime}$ ( $\left.3900.74 \mathrm{~kg} \mathrm{ha}^{-1}\right)$ in the $\mathrm{F}_{6}$ generation, 'Stoneville453' × 'Tamcot Sphinx-II' (3350.87 $\mathrm{kg} \mathrm{ha}^{-1}$ ) and 'Çukurova 1518 ' control variety $\left(3400.53 \mathrm{~kg} \mathrm{ha}^{-1}\right)$ were identified in the $\mathrm{F}_{7}$ generation. From the point of ginning percentage in the $\mathrm{F}_{6}$ and $\mathrm{F}_{7}$ generations, while the highest value was obtained from 'Maraş92' × 'Sayar314' (41.65\%$40.50 \%)$ and 'Carmen' variety ( $41.15 \%-40.40 \%)$, the lowest value was obtained from 'TamcotCD3H' $\times$ 'Tamcot Luxor-I’ (36.82\%-36.80\%) hybrid combination.

Results from the analysis of variance for fiber fineness, fiber length, and fiber strength are given in Tab. 3. In the analysis conducted pursuant to FF, FL and FS, difference among genotypes was significant at a level of 5\%. While the thinnest FF value was determined in 'Sayar314' $\times$ 'Stoneville453-I' (4.39 mic.) and thickest FF value was determined in 'Stoneville453' × 'Tamcot Sphinx' (5.04 mic.) and 'TamcotCD3H' × 'Tamcot Luxor-I' (5.02 mic.) in the $\mathrm{F}_{6}$ generation, while the thinnest FF value was obtained from 'Carmen' control variety ( $4.62 \mathrm{mic}$.) and 'Stoneville $453^{\prime} \times$ 'Tamcot Sphinx-I' (4.65 mic.) and thickest FF values were obtained from 'Stoneville 453' $\times$ 'Tamcot Sphinx' (5.24 mic.), 'Sayar 314' × 'Stoneville 453-II' (5.19 mic.) and 'Tamcot CD3H' $\times$ 'Tamcot Luxor-I' (5.15 mic.) in the $\mathrm{F}_{7}$ generations. While in terms of FL; the tallest fiber value was detected in 'Carmen' control variety $(31.44 \mathrm{~mm}$, $31.22 \mathrm{~mm})$ and 'Sayar314' $\times$ 'Stoneville453-I' $(31.11 \mathrm{~mm}$, $30,47 \mathrm{~mm}$ ) hybrid in both of F6 and F7 generations, the shortest fiber value was determined in 'Stoneville453' $\times$ 'Tamcot Sphinx-II' (28.13 mm) in the $\mathrm{F}_{6}$ generation and 'TamcotCD3H' × 'Tamcot Luxor-III' (27.98 mm) in $\mathrm{F}_{7}$ generations. According to the FS in the $\mathrm{F}_{6}$ generation, the highest value was detected in 'Maraş92' $\times$ 'Tamcot Sphinx'
(33.15 $\left.\mathrm{g} \mathrm{tex}^{-1}\right)$ and 'Sayar314' × 'Stoneville453-I' (32.70 g tex $\left.{ }^{-1}\right)$ and 'Carmen' variety $\left(32.65 \mathrm{~g} \mathrm{tex}^{-1}\right)$ and the lowest FS was detected in susceptible 'Cukurova 1518' control variety $\left(27.80 \mathrm{~g} \mathrm{tex}^{-1}\right)$. In the $\mathrm{F}_{7}$ generation, the highest values were detected in tolerant 'Carmen' variety $(33.40$ $\left.\mathrm{g} \mathrm{tex}^{-1}\right)$, 'Sayar314' $\times$ 'Stoneville453-I' (33.27 $\left.\mathrm{g} \mathrm{tex}^{-1}\right)$ and 'Maraş 92' × 'Sayar 314' (33.20 g tex $\left.^{-1}\right)$ and the lowest FS was detected in susceptible 'Çukurova 1518' control variety $\left(30.07 \mathrm{~g} \mathrm{tex}^{-1}\right)$ (Tab. 3).

\section{Discussion}

In pot experiments, DS values were higher than the values in field experiments because of making DS observations in seedling period (Tab. 1). Devey and Rosielle (1986) reported that disease observations in field were carried out in late period and disease observations in greenhouse was recorded in seedling period. Erdoğan et al. (2011) carried out a study in order to determine the reactions of 13 cotton genotypes to VW in greenhouse and field conditions they reported that DS values of greenhouse were higher than in field experiments. While genotypes in experiment were tolerant in terms of DS, they were located on the same group according to statistical analysis. Disease observations done in FDI, VDI, and PDI 'Carmen' (control) was detected as the most tolerant variety, similar values were obtained from 'TamcotCD3H' x 'Tamcot Luxor-III,' 'Maraş92' × 'Tamcot Sphinx' and 'Sayar314' x 'Stoneville453-I' breeding lines. The highest DS value was determined in susceptible 'Çukurova 1518' control variety (Tab. 1). The difference between experiments conducted in field and greenhouse came originate from the difference 
188

of susceptibility of G. hirsutum L. varieties to VW. El-Zik (1985), the resistance of Gossypium spp. showed difference to VW. In a study using five cotton genotype in greenhouse conditions. Cotton genotypes showed susceptibility at different level to VW (Corato et al., 2000). Erdoğan (2009) carried out study in 2005-2006 difference between variety and breeding lines were significant according to disease observations 'Carmen' control variety was the most tolerant variety in terms of disease severity. Bolek et al. (2011) reported that difference among genotypes was significant $(p \leq 0.01)$ as regard to variance analysis, the most tolerant genotype was 'Carmen', the most susceptible genotype was 'Çukurova 1518'. It was informed that 'Tamcot CD3H' and 'Tamcot HQ95' genotypes enhanced with MAR programme were highly resistant to VW, 'Tamcot Sphinx' and 'Tamcot Luxor' varieties were well resistant (Karademir, 2005).

'TamcotCD3H' $\times$ 'Tamcot Sphinx', 'Sayar314' $\times$ 'Stoneville453-I', 'Sayar314' $\times$ 'Stoneville453-II' and 'Stoneville453' $\times$ 'Tamcot Sphinx' breeding lines which were similar values with 'Carmen' control variety from the point of disease severity were the same group as statistical with 'Carmen' with regard to SCY. Also, the lowest SCY was detected in 'Çukurova 1518 ' control variety (Tab. 2). However, it was taken in count that not only the disease severity but also genotype, climate factors and cultural practices on SCY parameter. Bejarano-Alcazar et al. (1997) reported that total boll number, opening boll number and SCY decreased when foliar disease symptoms were seen before the first flowering period in cotton plants and the effect of disease on SCY after the first boll opening period in foliar symptoms. In addition to VW cultural practices and climate factors were also effective on SCY in infested cotton field throughout growing season (Hutmacher et al., 2005). In a study carried out in natural infested field and non-infested field it was determined that VW caused 15.93\% decrease on SCY (Erdoğan et al., 2006). SCY, lint yield and boll number $\left(\mathrm{m}^{2}\right)$ parameter values in resistant varieties to VW were higher than susceptible or tolerant genotypes (Jun-jie et al., 2010). Karademir et al. (2010) determined that varieties such as ST-453 and Deltaopal which are extremely susceptible to VW had also highly efficient for seed cotton yield. Arabsalmani et al. (2011) reported that VW decreased the SCY and boll number. According to GP it was determined that genotypes, which had high and low disease severity values, were statistically on the same group (Tab. 2). This variation in GP values was thought to originate from genotype $\mathrm{x}$ environment interactions rather than VW. Although, results obtain from experiments with regard to GP paralleled with the results of El-Zik (1985) and Erdoğan et al. (2006).

In this study, while 'Sayar314' × 'Stoneville453-I' similar disease severity values with tolerant 'Carmen' variety was determined as the thinnest fiber value, it was statistically on the same group which 'Carmen' variety in terms of FL and FS. Genotypes which had low and high disease severity values were statistically on the same group in terms of FL. This could be because of cultural practice and genotype $\times$ environment interactions rather than VW. While genotypes tolerant to $\mathrm{VW}$ had also high FS values, the lowest FS value was obtained from susceptible 'Cukurova 1518' control variety (Tab. 3). Simsek and Sahin (1980) and Yelin and Ersan (1985) reported that VW negatively affected fiber quality and besides fiber technological properties were not well in resistant varieties. Kechagia and Xanthpoulus (1998) and Azaddisfani and Zangi (2007) revealed that FL was effected fewer than VW, variation of FF was substantially dependent on varieties and effect VW on FS changed according to the varieties. Aguado et al. (2010) who reported that in natural infested disease differences among the genotypes were significant in variance analysis regarded with fiber characteristics. Especially the difference between FF and FL parameters were originated from genotype $\times$ environment interaction. Arabsalmani $e t$ al. (2011) who reported that VW decreased FF, FL and FS.

\section{Conclusions}

As a result, a major of genotypes developed were detected as tolerant as 'Carmen' variety to VW. At the same time, breeding lines were determined to be promising with regards to parameters such as SCY, GP and FS. By being transferred to the next generation, the performances of genotypes, tolerant to VW, yielding and having well fiber quality properties must be seen in macro plots. As is known, using breeding lines having new combinations obtained from genetically different parents will decrease the susceptibility to biotic and abiotic stress factors in breeding programs that will be formed.

\section{References}

Aguado A, De Los Santos B, Gamane D, Garci'a del Moral LF, Romero F (2010). Gene effects for cotton-fiber traits in cotton plant (Gossypium hirsutum L.) under Verticillium conditions. Field Crops Res 116:209-217.

Allen SJ, Lonergan PA (1998). The Development of New Strains of Verticillium dabliae in Australia. Proceedings of the World Cotton Research Conference-2, Athens- Greece, 923-926 p.

Anonymous (2011). Plant Production Statistical. Turkish Statistical Institute. (in Turkish with English abstract).

Arabsalmani M, Okhovat SM, Sharifitherani AM, Nikkha J, Safaie $N$ (2011). Epidemiology of verticillium wilt of cotton in golestan provience: Effect of verticillium wilt on quantitative and qualitative characters of cotton on yield. Iran J Plant Pathol 47(1):1-2.

Azaddisfani F, Zangi MR (2007). Verticillium Wilt Tolerance in Some Cotton Genotypes. Plant Pathol J 6(2):206-209.

Bejarano-Alcazar J, Blanco LMA, Melero V, Jimenez Diaz RM (1996). Etiology. Importance and distrubation of verticillium wilt of cotton in southern Spain. Plant Dis 80(11):1233- 
1238.

Bejarano-Alcazar J, Blanco LMA, Melero V, Jimenez Diaz RM (1997). The influence of verticillium wilt epidemics on cotton yield in southern Spain. Plant Pathol 46:168-178.

Bolek Y, Erdoğan O, Bardak A (2011). Response of some cotton genotypes to Verticillium Wilt (Verticillium dabliae Kleb) disease. $4^{\text {th }}$ Plant Protection Congress of Turkey, 349, Kahramanmaraş-Turkey. (in Turkish, with English abstract).

Buchenauer HD, Erwin C (1976). Effect of the plant growth retardant Pydanon on Verticillium wilt of cotton and tomato. Phytopathol 49:68-72.

Bugbee WM, Presley JT (1967). A rapid inoculation technique to evaluate the resistance of cotton to Verticillium alboatrum. Phytopathol 57:1264.

Corato U, Piscioneri I, Palazzo S, Orlandini S (2000). The wilting of cotton by Verticillium dabliae in Basilicata (Southern Italy). J Petria 10(2):77-84.

Devay JE (2001). Seedling Diseases, 13-14 p. In: Kirkpatrick TL, Rothrock CS (Eds.). Compendium of cotton diseases, Second ed., APS Pres VII+77.

Devey ME, Rosielle A (1986). Relationship between field and greenhouse ratings for tolerance to Verticillium Wilt on cotton. Crop Sci 26:1-4.

El-Zik KM (1985). Integrated control of Verticillium Wilt of cotton. Plant Dis 1025-1032.

Erdoğan O, Sezener V, Ozbek N, Bozbek T, Yavas I, Unay A (2006). The effects of Verticillium Wilt (Verticillium dabliae Kleb.) on cotton yield and fiber quality. Asian J Plant Sci 5(5):867-870.

Erdoğan O (2007). Effects of fluorescent pseudomonads on the control of Verticillium Wilt (Verticillium dabliae Kleb.) and plant growth of cotton. University of Adnan Menderes, Graduate School of Natural and Applied Sciences Department of Plant Protection Science, Ph.D Thesis, 121, AydinTurkey. (in Turkish, with English abstract).

Erdoğan O (2009). Determination of reactions of some cotton breeding lines against cotton wilt disease caused by Verticillium dahliae Kleb. Adnan Menderes University, Journal of The Faculty of Agriculture 6(2):9-16. (in Turkish, with English abstract).

Erdoğan O, Dundar H, Gore ME (2011). Determination of reactions of some cotton genotypes against verticillium wilt disease caused by Verticillium dahliae Kleb. Plant Protec Bull 51(2):159-173. (in Turkish, with English abstract).

Galanopoulo S (2006). http://www.Ressources.ciheam.org./ om/pdf/s14/CI01190.

Hanson LE (2000). Reduction of Verticillium Wilt symptoms in cotton following seed treatment with Trichoderma virens. The J of Cotton Sci 4:224-231.

Hutmacher B, Wright SR, Vargas D, Munk B, Marsh RM, Davis $S$ (2005). Field check, University of California cooperative extension. Cotton info, ucdavis.edu./mages/filed 520 check.
Joaquim TR, Rowe RC (1990). Reassessment of vegetative compatibility relationships among strains of Verticillium dahliae using nitrate-nonutilizing mutants. Phytopathol 80:1160-1166.

Jun-jie H, Qi-xiang M, Huan-min L, Xin-he J, Zhong-dong D, Shu-mei L, Xiao-wei C, Zhi-xin Z (2010). Effects of grafting cotton on Verticillium Wilt resistance, yield and fiber quality of cotton. Scientia Agricultura Sinica 19:3974-3980.

Karademir E (2005). Assessment of yield, earliness and fiber quality Properties in the F1 generations obtained crosses of multi adversity resistance (Mar) cotton varieties and regional varieties. Cukurova University, Graduate School of Natural and Applied Sciences Department of Field Crops Science, Ph. D Thesis, 140, Adana-Turkey. (in Turkish, with English abstract).

Karademir E, Karademir Ç, Ekinci R, Baran B, Sağır A (2010). Assessment of tolerance level of some cotton ( $G$. hirsutum L.) varieties against Verticillium Wilt ( $V$. dabliae Kleb.). Not Bot Horti Agrobo 38(1):196-202.

Karman M (1971). General information in plant protection investigations, evaluation and performing of the trials. Regional Agricultural Protection Institute, 279, Izmir-Turkey. (in Turkish, with English abstract)

Kechagia OE, Xanthopoulos FP (1998). Degree of Verticillium Wilt infestation and the relative damage in fibre quality parameters. World Cotton Research Conference-2, AthensGreece, 336 p.

Kurt S, Bicici M (1998). Development of $V$. dabliae in cotton plants grown in cukurova and reaction of some cultivars to Wilt. World Cotton Research Conference-2, AthensGreece, 919-922 p.

Mert M, Kurt S, Gencer O (2001). Determination of reaction and agricultural and technological properties on some cotton breeding line and varieties to Wilt disease (Verticillium dahliae Kleb.). $4^{\text {th }}$ Field Crops Congress of Turkey, TekirdagTurkey, 193-197 p. (in Turkish, with English abstract).

Simsek M, Sahin A (1980). Determination of resistance on cotton varieties to Wilt disease. Nazilli Cotton Research Institute 27:30, Aydin-Turkey. (in Turkish, with English abstract).

Unal M, Aydın G (1980). Resistance to Verticillium Wilt in Cotton Breeding. Nazilli Cotton Research Institute, 27:23, Aydin-Turkey. (in Turkish, with English abstract).

Wilhelm S, Sagen JE, Tietz H (1974). Resistance to Verticillium wilt in cotton: source, techniques of identification, inheritance trends and Resistance potential of Multipline Cultivars. Phytopathol 64:924-931.

Wu F, Wu FA (1997). Resistant response of the new upland cotton varieties to the defoliating strain of $V$. dabliae Kleb. China-Cotton 24(9):11-13.

Yelin D, Ersan K (1985). A research on yield and some technological characters and sensivity of cotton varieties to $V$. dahliae Kleb. in Kahramanmaraş. Journal of Turkish Phytopathol 14:96. 